\title{
Enriched expression of genes associated with autism spectrum disorders in human inhibitory neurons
}

\author{
Ping Wang ${ }^{1}$, Dejian Zhao ${ }^{1}$, Herbert M. Lachman ${ }^{1,2,3,4}$ and Deyou Zheng (1) 1,3,5
}

\begin{abstract}
Autism spectrum disorder (ASD) is highly heritable but genetically heterogeneous. The affected neural circuits and cell types remain unclear and may vary at different developmental stages. By analyzing multiple sets of human single cell transcriptome profiles, we found that ASD candidates showed relatively enriched gene expression in neurons, especially in inhibitory neurons. ASD candidates were also more likely to be the hubs of the co-expression gene module that is highly expressed in inhibitory neurons, a feature not detected for excitatory neurons. In addition, we found that upregulated genes in multiple ASD cortex samples were enriched with genes highly expressed in inhibitory neurons, suggesting a potential increase of inhibitory neurons and an imbalance in the ratio between excitatory and inhibitory neurons in ASD brains. Furthermore, the downstream targets of several ASD candidates, such as CHD8, EHMT1 and SATB2, also displayed enriched expression in inhibitory neurons. Taken together, our analyses of single cell transcriptomic data suggest that inhibitory neurons may be a major neuron subtype affected by the disruption of ASD gene networks, providing single cell functional evidence to support the excitatory/inhibitory (E/I) imbalance hypothesis.
\end{abstract}

\section{Introduction}

Autism spectrum disorder (ASD) is a class of neurodevelopmental disorders characterized by persistent deficits in social communication/interaction and restricted, repetitive patterns of behaviors, interests or activities $(\mathrm{DSM}-5)^{1}$. Recent epidemiology studies have reported that 1 in 68 children is diagnosed with ASD, with a 3 to 4fold increased risk for boys ${ }^{2,3}$. Family and twin studies have found that ASD is highly heritable ${ }^{4,5}$, but the genetic risk factors for ASD are highly heterogeneous and up to one thousand genes are estimated to be involved, with no single gene accounting for $>1-2 \%$ of the cases ${ }^{6}$. These ASD candidate genes converge on several molecular and cellular pathways, such as synaptic function, Wnt-signal

Correspondence: Deyou Zheng (deyou.zheng@einstein.yu.edu)

${ }^{1}$ Department of Genetics, Albert Einstein College of Medicine, 1300 Morris Park Ave., Bronx, NY, USA

${ }^{2}$ Department of Psychiatry and Behavioral Sciences, Albert Einstein College of Medicine, 1300 Morris Park Ave., Bronx, NY, USA

Full list of author information is available at the end of the article and chromatin remodeling ${ }^{7-12}$, indicating that ASD pathogenesis is a complicated multidimensional process modulated by genetic factors that play key roles in response to intrinsic developmental signaling and environmental perturbations.

At the cellular level, a human brain can be divided into distinct functional regions that are composed of diverse but densely connected cell types. It has been reported that ASD risk genes form co-expression networks that are expressed at relatively higher levels in specific embryonic prefrontal cortex regions and layers ${ }^{13,14}$, and ASD mutations could potentially affect certain brain areas and cell types more strongly than others ${ }^{15}$. For example, $\mathrm{Xu}$ et al. previously developed a method ("cell type-specific expression analysis") to analyze microarray gene expression data from mouse and human brains, including cell type data from translating ribosome affinity purification (TRAP) technology, and found that multiple cell types could be implicated in $\mathrm{ASD}^{16}$, e.g., astrocytes, glia and cortical interneurons. Subsequently, Zhang et al., also 
using TRAP data from mouse lines, observed that an expression signature shared by ASD risk genes is a strong and positive association with specific neurons in different brain regions, including cortical neurons ${ }^{17}$.

A limitation of these previous studies is related to the concern that the resolution of cell types may not be sufficient, in addition to other limitations specifically related to the microarray platform. This can be addressed by single cell RNA-seq (scRNA-seq) analysis that measures gene expression profiles for hundreds to thousands of cells in a tissue sample simultaneously, which can resolve cell types and reveal expression heterogeneity ${ }^{18}$. With a mouse scRNA-seq dataset ${ }^{19}$ and a novel computational method, Skene et al. suggested that genetic susceptibility of ASD primarily affected interneurons and pyramidal neurons ${ }^{20}$. The method is called "expression weighted cell-type enrichment" (EWCE), which evaluates statistically whether a set of genes shows higher expression in a particular cell type than what is expected by chance ${ }^{20}$. Skene et al recently also reported that pyramidal cells, medium spiny neurons and certain interneurons could be more important for schizophrenia than other brain cell types ${ }^{21}$.

While the above studies have suggested that ASD risk genes can have cell type specific functions and expression patterns, and some brain cell types may be more prone to the effects of ASD-associated mutations, no similar studies have been performed using human cell type-specific functional genomic data, especially scRNA-seq data. This is important because it has been shown that many gene expression modules are human specific, several of which are correlated with brain disorders, such as Alzheimer's disease $^{22}$, despite the extensive global network similarity of the human and mouse brain transcriptomes. Our previous study of the transcriptional regulatory network modulated by a neural master regulator, REST/NRSF, also found that ASD genes are enriched among human specific REST targets ${ }^{23}$. Moreover, the human brain is much more complex than the mouse brain, especially in some regions, such as the frontal and temporal lobes, which have undergone enormous changes during primate evolution ${ }^{24}$. More importantly, no systematic studies related to ASD have been carried out in which excitatory and inhibitory neuronal transcriptomes have been compared, despite the long-standing E/I imbalance hypothesis, which has been proposed as a model to explain some ASD-related behaviors $^{25-28}$. Therefore, to address if some cell types are more prone to genetic network disruptions potentially occurring in the brains of individuals with ASD, we have collected multiple human neural or brain expression datasets, most of which were derived from advanced scRNA-seq analysis, and evaluated if genes implicated in ASD show different expression profiles across human neural cell types. The gene sets in our study include a)
ASD candidate genes, b) differentially expressed genes between ASD individuals and controls, and c) downstream targets of several ASD candidates. We found that these genes consistently show significantly enriched expression in human neurons, particularly inhibitory neuron, suggesting that inhibitory neuron is the major cell type affected in ASD. This finding is consistent with the hypothesis that a disruption of the balance between inhibitory and excitatory signaling could be an important underlying mechanism of ASD pathogenesis.

\section{Materials and methods \\ Human single cell RNA-seq data}

Four sets of human scRNA-seq data were analyzed. For the fetal brain and cerebral organoid datasets ${ }^{29}$ and the adult brain dataset ${ }^{30}$, raw scRNA-seq reads were aligned to the human reference genome (GRCh37/hg19) using STAR (ver. 2.0.13) ${ }^{31}$. Duplicate reads were removed using Samtools (ver. 0.1.19) ${ }^{32,33}$. Gene length and uniquely mapped reads for each gene were calculated using featureCounts in subread package (ver. 1.4.6) ${ }^{34}$ with gene models from Ensembl release 74. Fragments per kilobase of transcript per million mapped reads (FPKM) values were calculated using $\mathrm{R}$ (https://www.r-project.org/) according to its definition. For the neuron subtype dataset for excitatory and inhibitory neurons, transcripts per kilobase million (TPMs) were obtained from the original paper $^{35}$. In all four cases, we used the authors' original classification of cell types.

\section{Lists of genes associated with ASD, schizophrenia and other brain disorders}

ASD candidate genes were downloaded from the SFARI database (https://gene.sfari.org/autdb/GS_Home.do; genes scored as high confidence, to minimal evidence and syndromic) and the AutismKB (core dataset) ${ }^{36}$. The two schizophrenia gene lists were from the SZgene database ${ }^{37}$ and a recent GWAS report ${ }^{38}$. Bipolar disorder associated genes were from the BDgene database ${ }^{39}$. Other gene lists associated with brain diseases were described in our previous publication ${ }^{23}$. Genes encoding excitatory and inhibitory postsynaptic density (PSD) proteins were from a previous study by Uezu et al. ${ }^{40}$ The genes associated with human height were from a previous $\mathrm{GWAS}^{41}$. Gene lists were provided in Table S1.

\section{Differentially expressed genes between ASD and controls}

Gene expression in postmortem cortices ("Cortex1") ${ }^{42}$ was used to detect differentially expressed genes by GEO2R (https://www.ncbi.nlm.nih.gov/geo/geo2r/), which is defined here as FDR $<0.05$ and fold change $>1.3$ -the same criteria as used in the original paper. Differentially expressed genes in blood ${ }^{43}$ were also detected by GEO2R and defined as $p<0.05$. Gene lists from other 
brain-related samples, including postmortem cortices ("Cortex2"44 and "Cortex3"45), induced pluripotent stem cell (iPSC)-derived cerebral organoids ("Organoid") ${ }^{46}$, neural progenitor cells (NPC) $)^{47}$, and neurons ("Neuron $1 " 47$ and "Neuron2" ${ }^{\text {" }}$ ), were obtained from the original papers and provided in Table S1.

\section{Downstream genes of ASD candidates}

CHD8-regulated genes in NPCs, neurons ${ }^{49}$ and cerebral organoids $^{50}$, CYFIP1-regulated genes ${ }^{51}$, TCF4 and EHMT1-regulated genes ${ }^{52}$, MBD5 and SATB2-regulated genes $^{53}$, NRXN1-regulated genes ${ }^{54}$ and ZNF804Aregulated genes ${ }^{55}$ were from studies where the expression of a known ASD candidate was reduced by knockout or knockdown. Gene lists were obtained from the original papers and provided in Table S1.

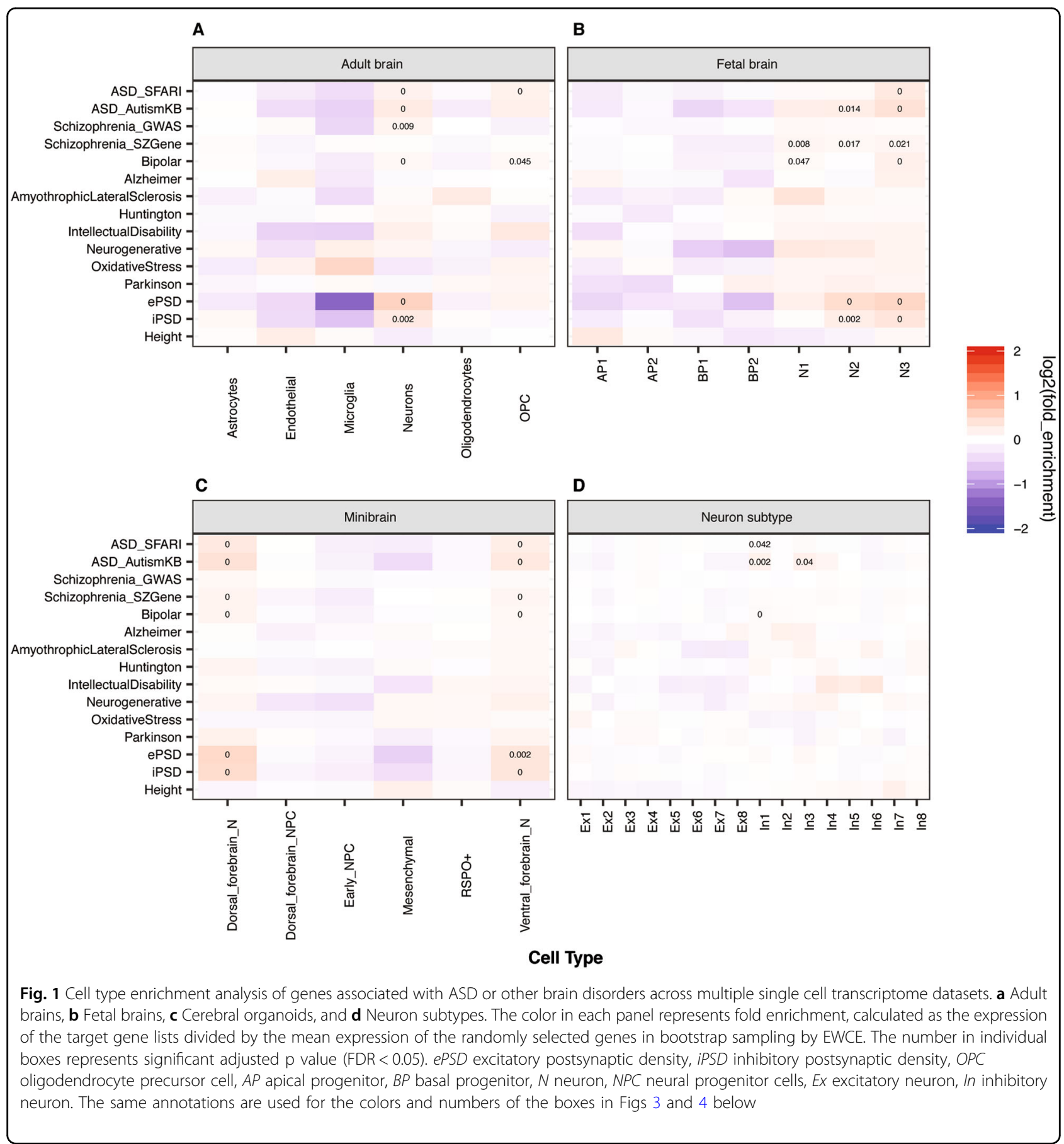




\section{EWCE analysis for determining enriched expression}

For each cell type, mean of $\log 2$ (FPKM or TPM) across all samples were calculated and imported into the EWCE $(\mathrm{v} 1.3 .0)^{20}$ to determine enriched expression. To determine specifically which genes contributed to the statistical significance of enriched expression, we used the "generate. bootstrap.plots" function in EWCE with 10,000 permutations to generate "mean bootstrap expression" (see Fig. 2 in the EWCE paper ${ }^{20}$ for details). We further defined genes whose relative expression was $>1.2$-fold greater than the mean bootstrap expression as "enriched genes". The resultant lists of enriched genes were analyzed for enriched gene ontology (FDR $<0.05)$ using the software goseq $^{56}$, in which the corresponding full gene list was used as background.

Weighted gene co-expression network analysis (WGCNA)

Signed co-expression networks were built using the WGCNA package ${ }^{57}$. The power of 18 was chosen, and blockwiseModules function was performed to build networks. Logistic regression was used to find modules expressed higher in excitatory or inhibitory neurons using eigengenes. P values were corrected by multiple testing to generate FDR. ToppGene ${ }^{58}$ was used to find Gene Ontology categories enriched in modules.

\section{Code availability}

The primary software EWCE (v1.3.0) ${ }^{20}$ was downloaded from https://www.bioconductor.org/packages/release/ bioc/html/EWCE.html. Additional codes for data processing are available from the authors upon request. An early version of this manuscript ${ }^{59}$ was submitted to bioRxiv before publication.

\section{Results}

\section{ASD candidate genes show enriched expression in} neurons, especially inhibitory neurons

It has generally been supposed that functional disruptions of a gene more likely affect the cells or tissues where the gene is highly expressed. Such a principle has often been used to support the discovery of risk genes from genetic studies in schizophrenia and $\mathrm{ASD}^{38,60}$. Accordingly, we have used the EWCE method to test what brain cell types are more likely to be affected by genes implicated in ASD, using transcriptomic data containing cell type identifies. Throughout this paper, the term "enrichment expression" or "enriched expression" in a particular cell type refers to a set of genes that have a higher level of expression within this cell type than expected by chance, as described in the EWCE method ${ }^{20}$. The method also accounts for a gene's overall expression across all cell types in a comparison, as the enrichment is actually computed based on relative expression values. We started with scRNA-seq expression data from six cell types from adult human brains (21-63 years old; a total of 285 cells), including neurons, microglia, and astrocytes (Fig. 1a) ${ }^{30}$. First, as a negative control, we found that genes associated with human height ${ }^{41}$ showed no enrichment of expression in any of the six cell types in test (Fig. 1). Conversely, as a positive control, genes encoding PSD proteins showed significant enrichment in neuron expression (Fig. 1).

Our analysis of the ASD candidates, obtained from either the SFARI (https://gene.sfari.org/autdb/GS_Home. do) or the AutismKB ${ }^{36}$, demonstrated that their expression was significantly enriched in human adult neurons and oligodendrocyte precursor cells but not astrocytes and microglia (Fig. 1a). Note that the significance of enrichment was a result of many but not few genes (Fig. S1), an observation applicable to results described below too (Table S2). As ASD is an early developmental disorder, we repeated the same analysis using a single cell transcriptome dataset from human fetal brains, including 226 single-cell transcriptomes from 12 and 13-wk postconception neocortex specimens ${ }^{29}$. The cell types in the fetal brain were classified differently from those in adult brains. We found that in comparison to apical and basal progenitors, ASD candidates were significantly enriched in neurons, especially mature neurons ("N2" and "N3") in fetal brains (Fig. 1b). We also found schizophrenia and bipolar disorder associated genes were similarly enriched in mature neurons (Fig. 1b), consistent with the known overlap of genetic risk factors among these disorders ${ }^{61}$. Meanwhile, genes associated with several other brain diseases, such as Alzheimer and Huntington, showed no significantly enriched expression in any of these cell types (Fig. 1b). Next, to study whether ASD candidates are enriched in neurons in specific brain regions, we analyzed single cell transcriptome data of cerebral organoids, including 495 single-cell transcriptomes ${ }^{29}$. Again, compared with NPCs, ASD candidates displayed significantly enriched expression in neurons - both dorsal and ventral forebrain neurons, as were schizophrenia and bipolar disorder associated genes (Fig. 1c). While not quite surprising, our analysis of these three cell type transcriptomic datasets showed that neurons, both early fetal neurons and adult neurons, are a major cell type potentially affected by ASD mutations, probably more so than neural progenitors. Finally, neurons could be largely classified into two major subtypes: excitatory and inhibitory neurons. Using scRNA-seq data of neuronal subtypes, whose classification was supported by known marker genes and including 3083 single-cell transcriptomes from six cortical regions of a control normal 51-year-old female postmortem brain ${ }^{35}$, we found that the expression of ASD candidates was significantly enriched in inhibitory neurons, especially among the subtypes "In1" and "In3" (Fig. 1d), which are superficial layer inhibitory neurons that originate from lateral ganglionic eminences ${ }^{35}$. These 
results suggest that functional disruptions of ASD genes as a group can affect inhibitory neurons more than excitatory neurons. Again, the significant enrichments were results from many genes (Fig. S2). For ASD genes, the expression "enriched genes" (see Methods) include neuronal markers, such as GAD1, RELN, VIP, as expected, and transcription regulators, such as CHD7, PAX6 and TBX1 (Table S2). Although it remains to be established with functional assays, this finding indicates that inhibitory neuron transcriptome dysregulation can occur in ASD brains, which is consistent with the E/I imbalance hypothesis in $\mathrm{ASD}^{25,62-65}$. GABAergic neurotransmission appears to play a role in both schizophrenia and bipolar disorder as well ${ }^{66,67}$. However, our results suggest that bipolar disorder but not schizophrenia-associated genes were significantly enriched among highly expressing genes in inhibitory neurons.

\section{ASD candidate genes are more likely to be hubs of co- expression modules in inhibitory neurons}

To further study the roles of ASD candidate genes in inhibitory neurons, we performed WGCNA to build a coexpression network from the neural subtype transcriptome data $^{35}$, resulting in 73 modules (Figure S3A and B). One of them showed high expression in excitatory neurons and contained 1936 genes, which were enriched for functions related to synaptic signaling, neuron projection and morphogenesis, as well as genes expressed in excitatory synapses (Figure S3,C). Another module contained 951 genes that were highly expressed in inhibitory neurons. They were enriched with genes involved in neurogenesis, positive regulation of synaptic transmission, and the GABA shunt (Figure S3,D). Consistent with the EWCE result, ASD candidates, from both the SFARI and AutismKB, were more significantly enriched in the module highly expressed in inhibitory neurons (odds ratio $(\mathrm{OR})=2.38, p=5.94 \mathrm{e}-07$, Fisher's exact test, one-tailed) than the module highly expressed in excitatory neurons $(\mathrm{OR}=1.42, p=0.018$, Fisher's exact test, one-tailed). Among the hub genes in the inhibitory module, nine were ASD candidates (Fig. 2a), including three genes encoding transcription factors $(A R X, D L X 2$, and $D L X 6)$ that are important for appropriate migration of inhibitory neurons to the $\operatorname{cortex}^{68}$, and three genes (SLC6A1, GAD1, $A L D H 5 A 1)$ that participate in GABA synthesis, release, reuptake and degradation, as described in the Reactome pathway $^{69}$. Notably, those ASD candidate genes had more connections in the inhibitory module than non-ASD candidates ( $p=0.0057$, Wilcoxon test; Fig. $2 \mathrm{~b}$ ), suggesting that ASD candidates tend to be the hubs in inhibitory module, and consequently, disease-associated mutations would likely lead to a disruption of the co-expression network. By comparison, in the excitatory module, ASD and non-ASD candidate genes had similar connections ( $p$ $=0.72$, Wilcoxon test; Fig. 2c, d).

\section{Genes up-regulated in ASD-derived neuronal samples show enrichment in inhibitory neurons}

Because of the extensive genetic heterogeneity in ASD, investigators have carried out transcriptomic studies in postmortem samples or ASD patient-derived neural samples with the goals of finding common pathways and cellular processes dysregulated in ASD brains or neural samples $^{42-48}$. We thus decided to study whether differentially expressed genes (DEGs) in molecular studies carried out between ASD and control subjects exhibited similar cell type-biased expression patterns as ASD candidate genes identified from genetic studies. We obtained DEGs in ASD brain or blood samples and analyzed their expression across brain cell types. Since, as shown above, we had uncovered the biased expression pattern of ASD candidate genes (from SFARI or AutismKB), we excluded them from the DEGs during EWCE analysis, in order to focus on downstream effects. In ASD cortex samples, upregulated genes were enriched with genes highly expressed in adult astrocytes and microglia (Fig. S4A), whereas down-regulated genes were enriched with genes highly expressed in neurons (Fig. S4B). This is consistent with previous reports ${ }^{42,44,45}$, but extends the finding to relatively mature neurons and both dorsal and ventral forebrain neurons (Fig. S4,B). We also found up-regulated genes in ASD cortex samples were enriched for highly expressed genes in NPCs (Fig. S4,A), a pattern not detected when ASD candidates were analyzed (Fig. 1). However, genes up-regulated in NPCs, neurons and cerebral organoids derived from ASD iPSC-lines showed enriched expression in neurons (Fig. S4,A), while downregulated genes in the patient-derived samples were enriched with genes expressed highly in astrocytes, microglia and NPCs (Fig. S4,B). These results suggest that cell types can be affected differently in early and late developing ASD brains. The difference may also reflect primary vs secondary effects. However, in our comparison of excitatory vs inhibitory neurons, we found that upregulated genes in both postmortem cortices and cerebral organoids were similarly enriched with genes highly expressed in inhibitory neurons (Fig. 3a). In a group of upregulated genes in ASD cortices (Cortex3_C1), enriched genes for In4 were enriched with regulation of cell motility; in upregulated genes in ASD-derived organoids (Organoid_TD11), enriched genes for In1 and In4 were enriched with DNA binding (Table S3). The downregulated genes from cortices and iPSC-derived neurons or cerebral organoids exhibited opposite enrichments, with the former enriched for high expression in excitatory and the latter in inhibitory neurons (Fig. 3b). Importantly, 


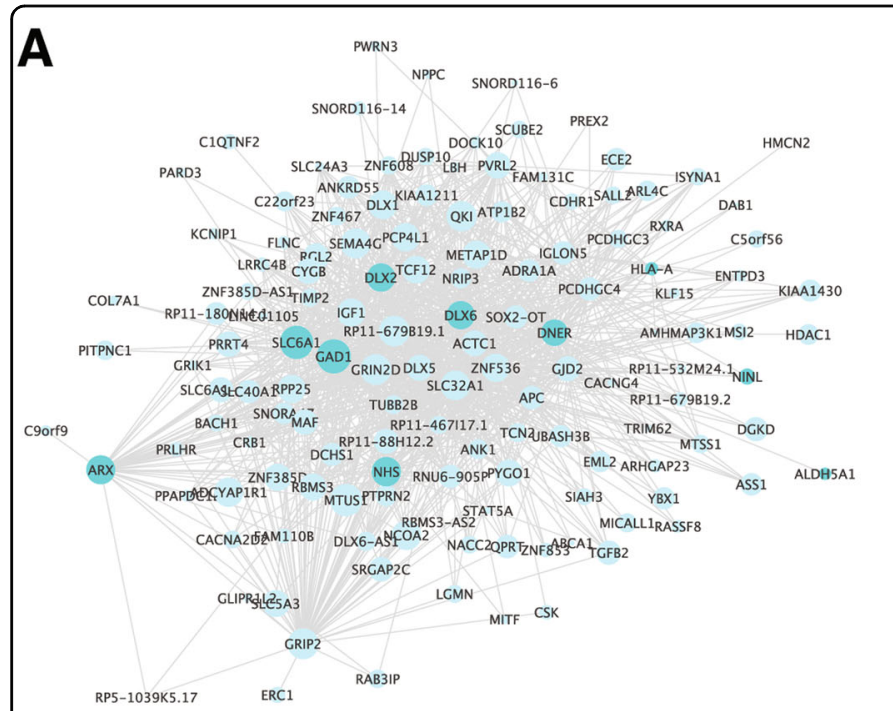

ASD genes

non-ASD genes

B

\section{Inhibitory module}

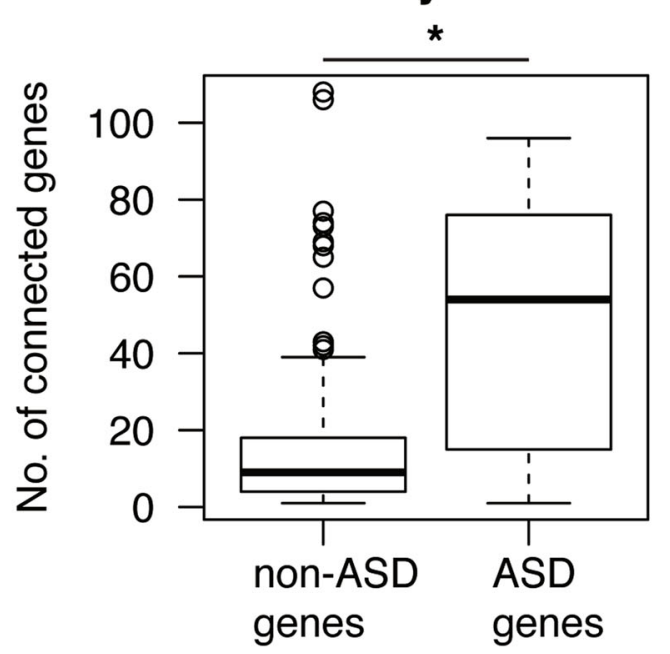

\section{C}

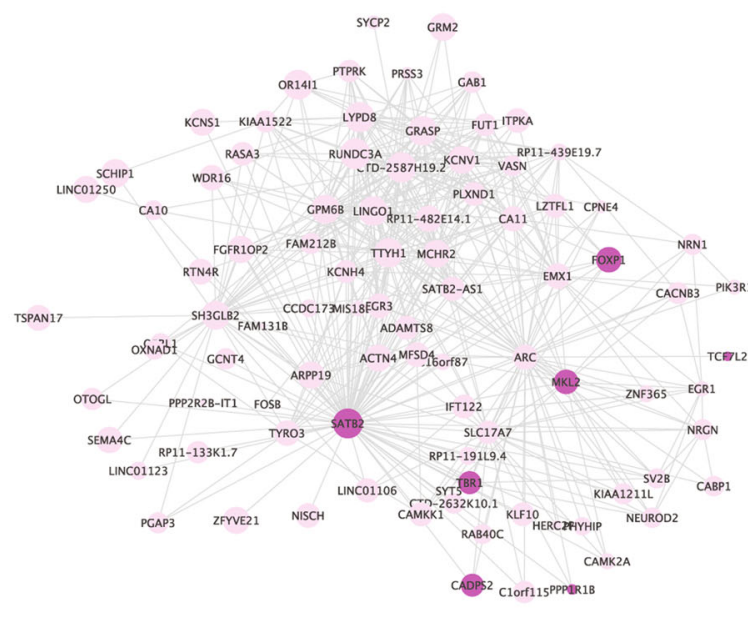

ASD genes

non-ASD genes

D

Excitatory module

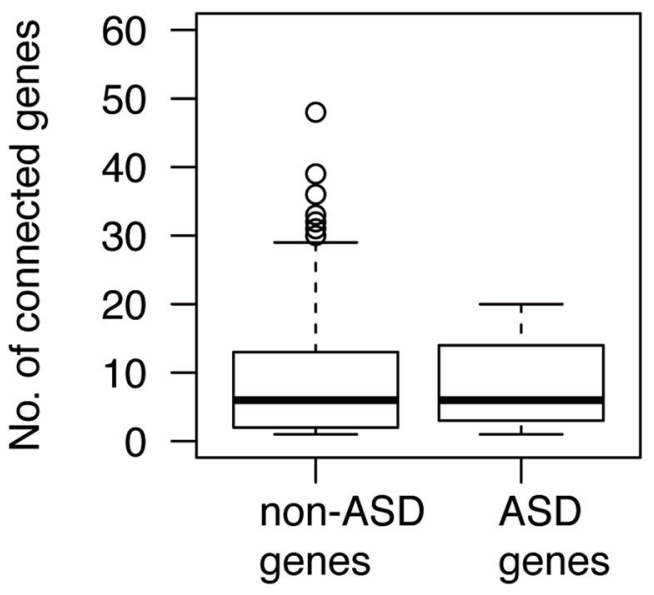

Fig. 2 Visualization of gene co-expression module associated with excitatory and inhibitory neurons. Genes with co-expression coefficient $>0.2$ from the WGCNA are shown as a network for $\mathbf{a}$ inhibitory and $\mathbf{c}$ excitatory modules. Node size represents the number of connected genes. Darker nodes are ASD candidate genes. Boxplots show the number of connections for ASD and non-ASD genes in inhibitory $\mathbf{b}$ and excitatory $\mathbf{d}$ modules. ${ }^{*} p<0.05$ Wilcoxon test

dysregulated genes in ASD blood samples ${ }^{43}$ did not exhibit any significant pattern of expression enrichment.

Downstream transcriptional targets of key ASD candidates are enriched among genes expressed highly in inhibitory neurons

Finally, we studied whether the downstream targets of ASD candidates genes show different expression enrichment patterns between inhibitory and excitatory neurons by analyzing the DEGs in human neural samples in which the expression of several top ASD (or schizophrenia) candidate genes have been reduced by either knockout or knockdown. We found that CHD8, EHMT1 and SATB2 regulated genes were exclusively enriched in inhibitory neurons (Fig. 4). For CHD8 regulated genes in iPSC derived neurons, expressionally enriched genes for $\operatorname{In} 1 / 2$ / 3/8 were functionally enriched for single-multicellular organism process; for SATB2 regulated genes, enriched 

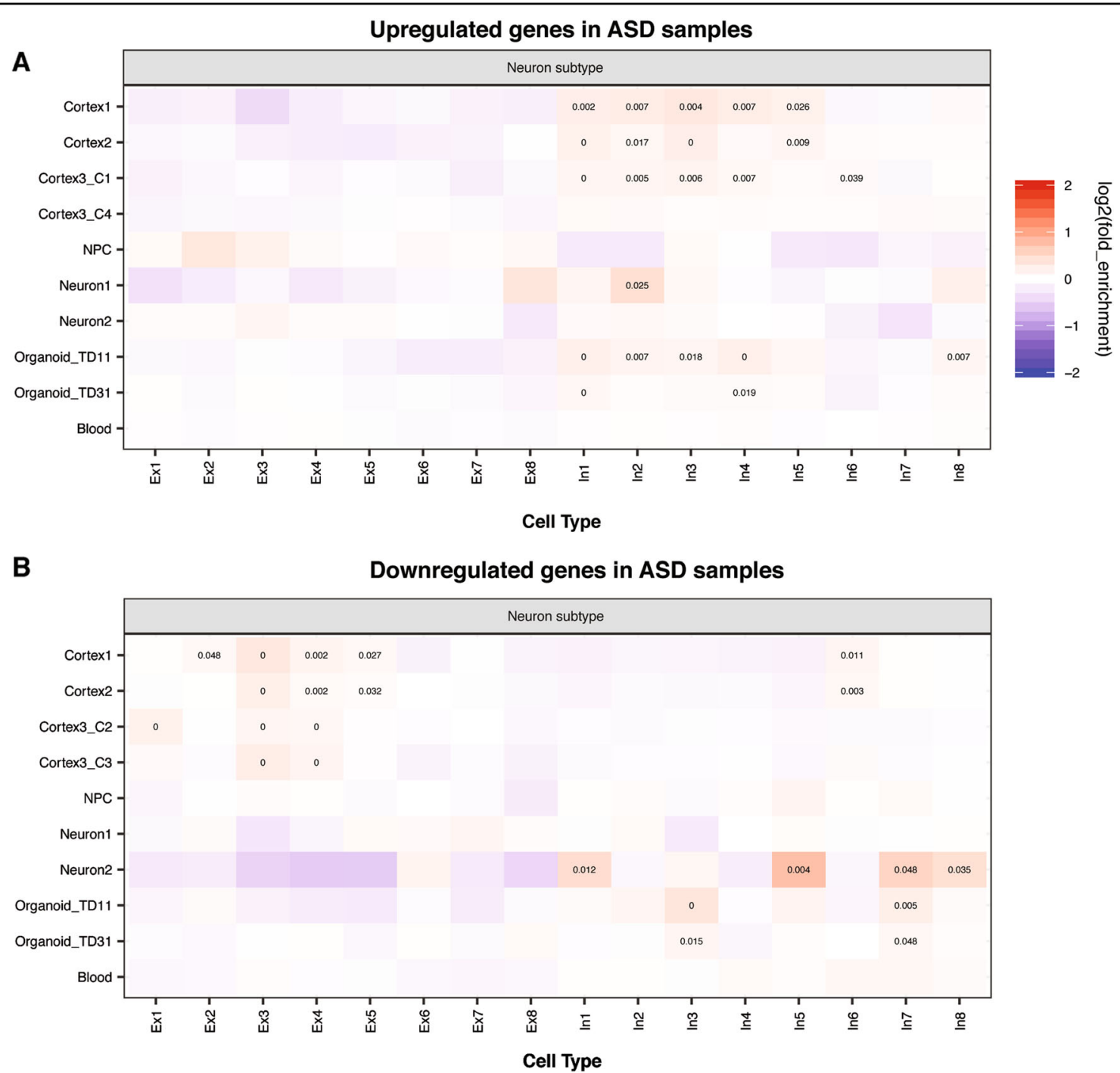

Fig. 3 Cell type enrichment analysis of upregulated $\mathbf{a}$ and downregulated $\mathbf{b}$ genes in ASD samples. Note that ASD candidates from SFARI or AutismKB have been excluded from DEGs

genes were enriched with embryonic organ development; for CYFIP1 regulated genes, enriched genes for In2 and In 4 are enriched with cell differentiation and locomotion, respectively (Table S3). Moreover, a general enrichment in inhibitory neuronal genes, especially those in "In $1 / 2 / 3$ " classes, was found among the targets of ASD candidates (Fig. 4). Among the downstream targets, DLX1, a transcription factor critical for inhibitory neuron function is markedly upregulated in ASD patient-derived telencephalic organoids ${ }^{46}$ and CHD8 knockout cerebral organoids $^{50}$, but $G A D 1$, an inhibitory neuron marker, was downregulated in SATB2 knockdown samples ${ }^{53}$. We analyzed DEGs from CYFIP1 knockdown in NPCs derived from three independent iPSC-lines and found both common and distinct enriched expression patterns. DEGs from two lines ( $\mathrm{C} 2$ and $\mathrm{C} 5)$ were enriched in inhibitory neurons, but C4 DEGs showed enriched expression in excitatory neurons (Fig. 4). This difference could reflect the limited overlap of the DEGs ${ }^{51}$, but also suggests an intriguing possibility that $\mathrm{E} / \mathrm{I}$ imbalances could be affected by inter-individual differences in genetic background. We should point out that $C H D 8$ and EHMT1 are expressed at a similar level in excitatory and inhibitory neurons, but $S A T B 2$ is expressed at a higher level in excitatory neurons. These findings further suggest that some ASD genes can affect the expression of key genes important for inhibitory and excitatory neurons and their targets may be involved 


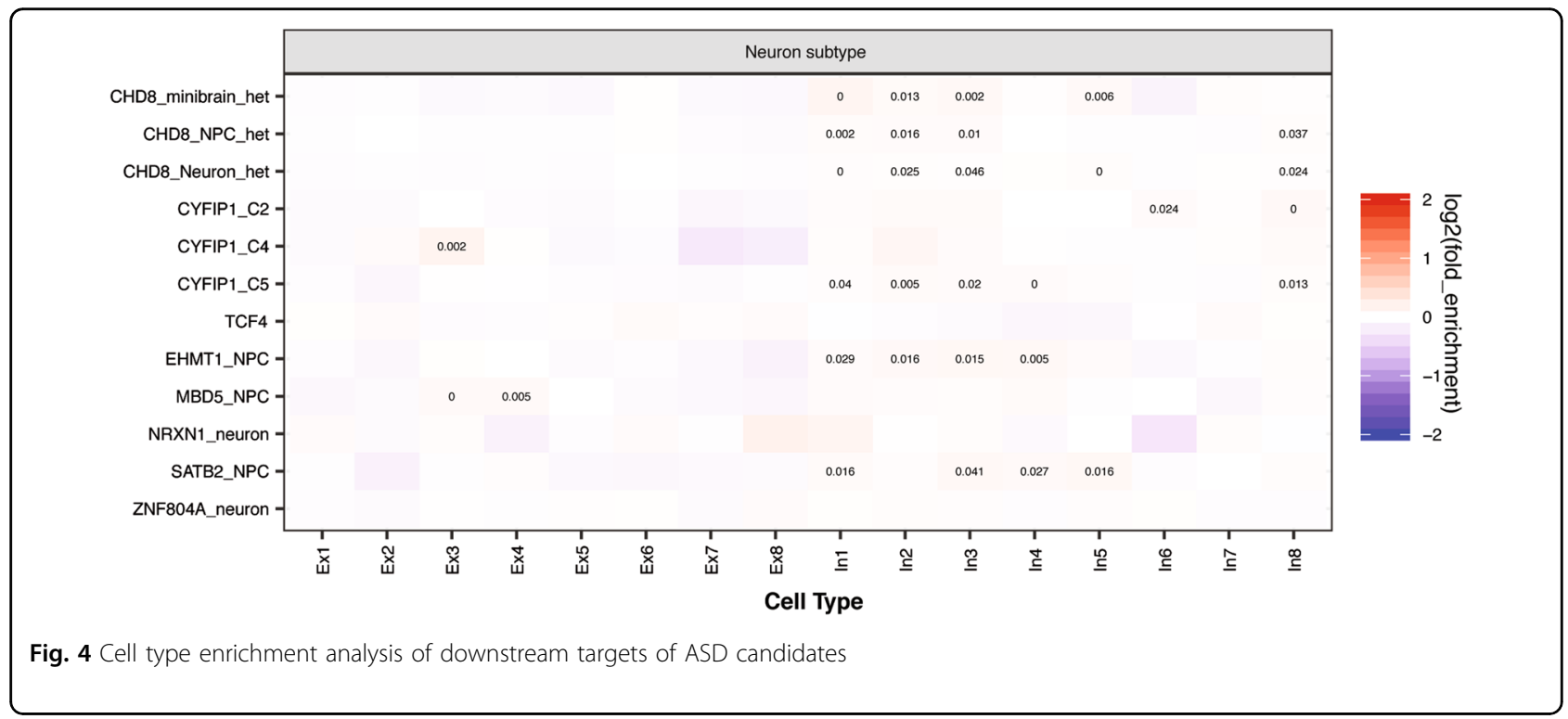

in the interaction or signaling balance between the two types of neurons.

\section{Discussion}

By integrating ASD candidates, dysregulated genes in ASD samples and downstream targets of ASD candidates with recently published human scRNA-seq datasets, we found that ASD-associated genes exhibited enriched expression in neurons, especially inhibitory neurons, with some developmental stage differences. The enrichment of inhibitory neuronal expression among ASD candidate genes provides molecular support for the finding that deficits in inhibitory neuronal function occurs in some syndromes with autism-associated behaviors, such as individuals with $A R X$ mutations ${ }^{70,71}$, Dravet syndrome caused by loss-of-function mutations in $S C N 1 A^{72}$, and Tuberous Sclerosis caused by mutations in TSC1/2 ${ }^{73,74}$ (for review, see ref. ${ }^{75}$ ). Our current findings are in line with the long-standing hypothesis that E/I signaling imbalance contributes to ASD. The attractive theory of an increase in the ratio between excitatory and inhibitory signaling provides a plausible explanation for the relative reduction in GABAergic signaling found in patients with ASD and their propensity to develop epilepsy ${ }^{75}$. However, a relative excess of inhibitory neuronal activity has been observed in mouse models of Rett Syndrome ${ }^{76}$, and mice with a targeted Mecp2 deletion restricted to GABAergic inhibitory neurons recapitulates most of the ASD-like features observed in animal models ${ }^{77}$, while restoring Mecp 2 expression reverses some of the phenotypical defects $^{78,79}$.

Our analysis showed enriched expression in inhibitory neurons for upregulated but not down-regulated genes in ASD samples. This seems inconsistent with the enriched expression of ASD candidates in inhibitory neurons, assuming their mutations lead to reduced expression and functional loss. One possibility is that some ASD candidates may function as transcriptional inhibitors or the abnormal expression of some ASD candidates could lead to an increase in the number of inhibitory neurons, in a subset of ASD subjects or in certain brain regions, perhaps as a compensation mechanism for a reduction of GABA receptors (or GABAergic function) in individual inhibitory neurons ${ }^{62}$. However, previous studies have reported an overproduction of GABAergic inhibitory neurons in ASD iPSC-derived organoids ${ }^{46}$ and neural cells ${ }^{47}$, with the former likely resulting from increased FOXG1 expression ${ }^{46}$, suggesting that an increase in inhibitory interneuron function could be due to a direct effect of some candidate genes. Another key transcription factor in GABAergic interneuron differentiation, $D L X 1$, was also upregulated in CHD8 knockout NPCs, neurons ${ }^{49}$, and cerebral organoids ${ }^{50}$. Furthermore, our study indicates that both primary and secondary ASD-affected genes may play roles in inhibitory neurogenesis and function, contributing to ASD pathogenesis. We should note that when, where and how an E/I imbalance contributes to ASD is unclear and certainly beyond the scope of the current study. Nevertheless, it is conceivable that E/I imbalance may tilt to one direction in a subset of ASD but to the other in a different subset.

Since neuronal subtype transcriptomes used in the current study were from an adult female brain ${ }^{35}$, and there are significant transcriptional (and structural) differences in the brain between the pre- to post-natal period, and from the teenage to adult stage ${ }^{80}$, it would be interesting to perform a similar EWCE study using scRNA-seq data from prenatal or fetal neurons in 
multiple brain regions from both sexes. Considering our findings, it is interesting to note that drugs targeting inhibitory neuron function are being developed to treat $\mathrm{ASD}^{81}$. Consequently, it would be valuable to study their effects in early and late developing brains, animal models, iPSC models, and in ASD subjects using brain imaging and electrophysiology to fully explore the therapeutic potential of such drugs.

Finally, we found that upregulated genes in postmortem ASD brains were enriched in microglia and astrocytes, which is consistent with original reports based on the mouse transcriptome ${ }^{42,44}$. This is consistent with the findings that activated microglia and astrocytosis occur in multiple brain regions of ASD patients ${ }^{82,83}$. However, ASD candidate themselves did not show such an enrichment in our analysis. Thus, dysregulation of neuron-glia signaling might be a secondary process in response to the initial insults elicited by the primary casual genetic variants, a testable hypothesis.

\section{Acknowledgements}

This study is supported by NIH grants (MH099427 to HL and HL133120 to DZ). We thank the High Performance Computing division at the Albert Einstein College of Medicine for computing support. We also acknowledge supports by the Albert Einstein College of Medicine's Rose F. Kennedy Intellectual and Developmental Disabilities Research Center and by the National Institutes of Health's National Institute of Child Health and Human Development (NIH/ NICHD) grant P30 HD071593.

\section{Author details}

${ }^{1}$ Department of Genetics, Albert Einstein College of Medicine, 1300 Morris Park Ave., Bronx, NY, USA. ²Department of Psychiatry and Behavioral Sciences, Albert Einstein College of Medicine, 1300 Morris Park Ave., Bronx, NY, USA. ${ }^{3}$ Department of Neuroscience, Albert Einstein College of Medicine, 1300 Morris Park Ave., Bronx, NY, USA. ${ }^{4}$ Department of Medicine, Albert Einstein College of Medicine, 1300 Morris Park Ave., Bronx, NY, USA. ${ }^{5}$ Department of Neurology, Albert Einstein College of Medicine, 1300 Morris Park Ave., Bronx, NY, USA

\section{Competing interests}

The authors declare that they have no competing financial interest.

Publisher's note: Springer Nature remains neutral with regard to jurisdictional claims in published maps and institutional affiliations.

\section{Supplementary information}

The online version of this article (doi:10.1038/s41398-017-0058-6) contains supplementary material.

Received: 26 May 2017 Revised: 13 August 2017 Accepted: 9 October 2017 Published online: 10 January 2018

\section{References}

1. Wingate M. et al. Prevalence of Autism Spectrum Disorder Among Children Aged 8 Years - Autism and Developmental Disabilities Monitoring Network, 11 Sites, United States, 2010. Mmwr Surveill Summ 63, (2014).

2. Developmental Disabilities Monitoring Network Surveillance Year Principal I, Centers for Disease C, Prevention. Prevalence of autism spectrum disorder among children aged 8 years - autism and developmental disabilities monitoring network, 11 sites, United States, 2010. MMWR. Surveill. Summ. 63, 1-21 (2014).
3. Loomes, R., Hull, L. \& Mandy, W. P. L. What Is the Male-to-Female Ratio in Autism Spectrum Disorder? A Systematic Review and Meta-Analysis. J. Am. Acad. Child. Adolesc. Psychiatr. 56, 466-474 (2017).

4. Sandin, S. et al. The familial risk of autism. JAMA. 311, 1770-1777 (2014).

5. Folstein, S. \& Rutter, M. Genetic influences and infantile autism. Nature. 265, 726-728 (1977).

6. De Rubeis, S. \& Buxbaum, J. D. Genetics and genomics of autism spectrum disorder: embracing complexity. Hum. Mol. Genet. 24, R24-R31 (2015).

7. Krumm, N., O'Roak, B. J., Shendure, J. \& Eichler, E. E. A de novo convergence of autism genetics and molecular neuroscience. Trends. Neurosci. 37, 95-105 (2014).

8. de la Torre-Ubieta, L., Won, H., Stein, J. L. \& Geschwind, D. H. Advancing the understanding of autism disease mechanisms through genetics. Nat. Med. 22, 345-361 (2016)

9. Chen, J. A., Penagarikano, O., Belgard, T. G., Swarup, V. \& Geschwind, D. H. The emerging picture of autism spectrum disorder: genetics and pathology. Annu. Rev. Pathol. 10, 111-144 (2015)

10. Willsey, A. J. \& State, M. W. Autism spectrum disorders: from genes to neurobiology. Curr. Opin. Neurobiol. 30, 92-99 (2015).

11. Jeste, S. S. \& Geschwind, D. H. Disentangling the heterogeneity of autism spectrum disorder through genetic findings. Nat Rev Neurol 10, 74-81 (2014)

12. Huguet, G., Ey, E. \& Bourgeron, T. The genetic landscapes of autism spectrum disorders. Annu. Rev. Genomics. Hum. Genet. 14, 191-213 (2013).

13. Willsey, A. J. et al. Coexpression networks implicate human midfetal deep cortical projection neurons in the pathogenesis of autism. Cell 155, 997-1007 (2013).

14. Parikshak, N. N. et al. Integrative functional genomic analyses implicate specific molecular pathways and circuits in autism. Cell 155, 1008-1021 (2013).

15. Chang, J., Gilman, S. R., Chiang, A. H., Sanders, S. J. \& Vitkup, D. Genotype to phenotype relationships in autism spectrum disorders. Nat. Neurosci. 18, 191-198 (2015)

16. Xu, X., Wells, A. B., O'Brien, D. R., Nehorai, A. \& Dougherty, J. D. Cell type-specific expression analysis to identify putative cellular mechanisms for neurogenetic disorders. J. Neurosci. 34, 1420-1431 (2014).

17. Zhang, C. \& Shen, Y. A Cell Type-Specific Expression Signature Predicts Haploinsufficient Autism-Susceptibility Genes. Hum. Mutat. 38, 204-215 (2017).

18. Stegle, O., Teichmann, S. A. \& Marioni, J. C. Computational and analytical challenges in single-cell transcriptomics. Nat. Rev. Genet. 16, 133-145 (2015).

19. Zeisel, A. et al. Brain structure. Cell types in the mouse cortex and hippocampus revealed by single-cell RNA-seq. Science 347, 1138-1142 (2015).

20. Skene, N. G. \& Grant, S. G. Identification of Vulnerable Cell Types in Major Brain Disorders Using Single Cell Transcriptomes and Expression Weighted Cell Type Enrichment. Front Neurosci 10, 16 (2016).

21. Skene, N. G. et al. Genetic Identification Of Brain Cell Types Underlying Schizophrenia. bioRxiv 2017. doi:https://doi.org/10.1101/145466

22. Miller, J. A., Horvath, S. \& Geschwind, D. H. Divergence of human and mouse brain transcriptome highlights Alzheimer disease pathways. Proc Natl Acad Sci U S A 107, 12698-12703 (2010).

23. Rockowitz, S. \& Zheng, D. Significant expansion of the REST/NRSF cistrome in human versus mouse embryonic stem cells: potential implications for neural development. Nucleic. Acids. Res. 43, 5730-5743 (2015).

24. Geschwind, D. H. \& Rakic, P. Cortical evolution: judge the brain by its cover Neuron. 80, 633-647 (2013).

25. Rubenstein, J. L. \& Merzenich, M. M. Model of autism: increased ratio of excitation/inhibition in key neural systems. Genes. Brain. Behav. 2, 255-267 (2003).

26. Gibson, J. R., Bartley, A. F., Hays, S. A. \& Huber, K. M. Imbalance of neocortical excitation and inhibition and altered UP states reflect network hyperexcitability in the mouse model of fragile $X$ syndrome. J. Neurophysiol. 100, 2615-2626 (2008)

27. Robertson, C. E., Ratai, E. M. \& Kanwisher, N. Reduced GABAergic Action in the Autistic Brain. Curr. Biol. 26, 80-85 (2016).

28. Bozzi Y., Provenzano G., Casarosa S. Neurobiological bases of autism-epilepsy comorbidity: a focus on excitation/inhibition imbalance. Eur J Neurosci (2017) doi:10.1111/ejn.13595.

29. Camp, J. G. et al. Human cerebral organoids recapitulate gene expression programs of fetal neocortex development. Proc Natl Acad Sci U S A 112, 15672-15677 (2015).

30. Darmanis, S. et al. A survey of human brain transcriptome diversity at the single cell level. Proc Natl Acad Sci U S A 112, 7285-7290 (2015). 
31. Dobin, A. et al. STAR: ultrafast universal RNA-seq aligner. Bioinformatics. 29, 15-21 (2013)

32. Li, H. A statistical framework for SNP calling, mutation discovery, association mapping and population genetical parameter estimation from sequencing data. Bioinformatics. 27, 2987-2993 (2011).

33. Li, H. et al. The Sequence Alignment/Map format and SAMtools. Bioinformatics. 25, 2078-2079 (2009).

34. Liao, Y., Smyth, G. K. \& Shi, W. featureCounts: an efficient general purpose program for assigning sequence reads to genomic features. Bioinformatics. $\mathbf{3 0}$, 923-930 (2014)

35. Lake, B. B. et al. Neuronal subtypes and diversity revealed by single-nucleus RNA sequencing of the human brain. Science 352, 1586-1590 (2016).

36. Xu, L. M. et al. AutismKB: an evidence-based knowledgebase of autism genetics. Nucleic. Acids. Res. 40, D1016-D1022 (2012). Database issue.

37. Allen, N. C. et al. Systematic meta-analyses and field synopsis of genetic association studies in schizophrenia: the SzGene database. Nat. Genet. $\mathbf{4 0}$ 827-834 (2008)

38. Schizophrenia Working Group of the Psychiatric Genomics C. Biological insights from 108 schizophrenia-associated genetic loci. Nature. 511, 421-427 (2014).

39. Chang, S. H. et al. BDgene: a genetic database for bipolar disorder and its overlap with schizophrenia and major depressive disorder. Biol. Psychiatr. 74, 727-733 (2013).

40. Uezu, A. et al. Identification of an elaborate complex mediating postsynaptic inhibition. Science 353, 1123-1129 (2016).

41. Lango Allen, $\mathrm{H}$. et al. Hundreds of variants clustered in genomic loci and biological pathways affect human height. Nature. 467, 832-838 (2010).

42. Voineagu, l. et al. Transcriptomic analysis of autistic brain reveals convergent molecular pathology. Nature. 474, 380-384 (2011).

43. Pramparo, T. et al. Cell cycle networks link gene expression dysregulation, mutation, and brain maldevelopment in autistic toddlers. Mol. Syst. Biol. 11, 841 (2015).

44. Parikshak, N. N. et al. Genome-wide changes in IncRNA, splicing, and regional gene expression patterns in autism. Nature. 540, 423-427 (2016).

45. Liu, X. et al. Disruption of an Evolutionarily Novel Synaptic Expression Pattern in Autism. PLoS Biol. 14, e1002558 (2016).

46. Mariani, J. et al. FOXG1-Dependent Dysregulation of GABA/Glutamate Neuron Differentiation in Autism Spectrum Disorders. Cell 162, 375-390 (2015).

47. Marchetto M. C. et al. Altered proliferation and networks in neural cells derived from idiopathic autistic individuals. Mol Psychiatr 22, 820-835 (2017)

48. Liu X. et al. Idiopathic Autism: Cellular and Molecular Phenotypes in Pluripotent Stem Cell-Derived Neurons. Mol Neurobiol 54, 4507-4523 (2017).

49. Wang, P. et al. CRISPR/Cas9-mediated heterozygous knockout of the autism gene CHD8 and characterization of its transcriptional networks in neurodevelopment. Mol Autism 6, 55 (2015).

50. Wang, P. et al. CRISPR/Cas9-mediated heterozygous knockout of the autism gene CHD8 and characterization of its transcriptional networks in cerebral organoids derived from iPS cells. Mol Autism 8, 11 (2017).

51. Nebel, R. A. et al. Reduced CYFIP1 in Human Neural Progenitors Results in Dysregulation of Schizophrenia and Epilepsy Gene Networks. PLOS. ONE. 11, e0148039 (2016)

52. Chen, E. S. et al. Molecular convergence of neurodevelopmental disorders. Am. J. Hum. Genet. 95, 490-508 (2014).

53. Gigek, C. O. et al. A molecular model for neurodevelopmental disorders. Trans/ Psychiatr 5, e565 (2015).

54. Zeng, L. et al. Functional impacts of NRXN1 knockdown on neurodevelopment in stem cell models. PLOS. ONE. 8, e59685 (2013).

55. Chen, J. et al. ZNF804A Transcriptional Networks in Differentiating Neurons Derived from Induced Pluripotent Stem Cells of Human Origin. PLOS. ONE. 10, e0124597 (2015).

56. Young, M. D., Wakefield, M. J., Smyth, G. K. \& Oshlack, A. Gene ontology analysis for RNA-seq: accounting for selection bias. Genome. Biol. 11, R14 (2010).

57. Langfelder, P. \& Horvath, S. WGCNA: an R package for weighted correlation network analysis. BMC. Bioinformatics. 9, 559 (2008).
58. Chen, J., Xu, H., Aronow, B. J. \& Jegga, A. G. Improved human disease candidate gene prioritization using mouse phenotype. BMC. Bioinformatics. 8, 392 (2007).

59. Wang P., Zhao D., Lachman H., Zheng D. Enriched expression of genes associated with autism spectrum disorders in human inhibitory neurons. bioRxiv 2017. doi: https://doi.org/10.1101/142968

60. Iossifov, I. et al. The contribution of de novo coding mutations to autism spectrum disorder. Nature. 515, 216-221 (2014).

61. Forstner, A. J. et al. Identification of shared risk loci and pathways for bipolar disorder and schizophrenia. PLOS. ONE. 12, e0171595 (2017).

62. Coghlan, S. et al. GABA system dysfunction in autism and related disorders: from synapse to symptoms. Neurosci. Biobehav. Rev. 36, 2044-2055 (2012).

63. Pizzarelli, R. \& Cherubini, E. Alterations of GABAergic signaling in autism spectrum disorders. Neural. Plast. 2011, 297153 (2011).

64. Gogolla, N. et al. Common circuit defect of excitatory-inhibitory balance in mouse models of autism. J Neurodev Disord 1, 172-181 (2009).

65. Vattikuti, S. \& Chow, C. C. A computational model for cerebral cortical dysfunction in autism spectrum disorders. Biol. Psychiatr. 67, 672-678 (2010).

66. Benes, F. M. \& Berretta, S. GABAergic interneurons: implications for understanding schizophrenia and bipolar disorder. Neuropsychopharmacology. 25, 1-27 (2001).

67. Belforte, J. E. et al. Postnatal NMDA receptor ablation in corticolimbic interneurons confers schizophrenia-like phenotypes. Nat. Neurosci. 13, 76-83 (2010).

68. Kelsom, C. \& Lu, W. Development and specification of GABAergic cortical interneurons. Cell Biosci 3, 19 (2013).

69. Croft, D. et al. The Reactome pathway knowledgebase. Nucleic. Acids. Res. 42 D472-D477 (2014). Database issue.

70. Olivetti, P. R. \& Noebels, J. L. Interneuron, interrupted: molecular pathogenesis of ARX mutations and X-linked infantile spasms. Curr. Opin. Neurobiol. 22, 859-865 (2012).

71. Shoubridge, C., Fullston, T. \& Gecz, J. ARX spectrum disorders: making inroads into the molecular pathology. Hum. Mutat. 31, 889-900 (2010).

72. Oakley, J. C., Kalume, F. \& Catterall, W. A. Insights into pathophysiology and therapy from a mouse model of Dravet syndrome. Epilepsia. 52, 59-61 (2011) Suppl 2.

73. Lasarge, C. L. \& Danzer, S. C. Mechanisms regulating neuronal excitability and seizure development following mTOR pathway hyperactivation. Front $\mathrm{Mol}$ Neurosci 7, 18 (2014).

74. Crino, P. B. Evolving neurobiology of tuberous sclerosis complex. Acta. Neuropathol. 125, 317-332 (2013).

75. Nelson, S. B. \& Valakh, V. Excitatory/Inhibitory Balance and Circuit Homeostasis in Autism Spectrum Disorders. Neuron. 87, 684-698 (2015).

76. Dani, V. S. et al. Reduced cortical activity due to a shift in the balance between excitation and inhibition in a mouse model of Rett syndrome. Proc Natl Acad Sci U S A 102, 12560-12565 (2005).

77. Chao, H. T. et al. Dysfunction in GABA signalling mediates autism-like stereotypies and Rett syndrome phenotypes. Nature. 468, 263-269 (2010).

78. Meng X. et al. Manipulations of MeCP2 in glutamatergic neurons highlight their contributions to Rett and other neurological disorders. elife 5, e14199 (2016).

79. Ure K. et al. Restoration of Mecp2 expression in GABAergic neurons is sufficient to rescue multiple disease features in a mouse model of Rett syndrome. elife 5 , e14198 (2016).

80. Colantuoni, $\mathrm{C}$. et al. Temporal dynamics and genetic control of transcription in the human prefrontal cortex. Nature. 478, 519-523 (2011)

81. Braat, S. \& Kooy, R. F. The GABAA Receptor as a Therapeutic Target for Neurodevelopmental Disorders. Neuron. 86, 1119-1130 (2015).

82. Morgan, J. T. et al. Microglial activation and increased microglial density observed in the dorsolateral prefrontal cortex in autism. Biol. Psychiatr. 68 , 368-376 (2010).

83. Vargas, D. L., Nascimbene, C., Krishnan, C., Zimmerman, A. W. \& Pardo, C. A Neuroglial activation and neuroinflammation in the brain of patients with autism. Ann. Neurol. 57, 67-81 (2005). 